\title{
Augmentation des capacités d'exportation de la raffinerie de Limbé au Cameroun
}

\author{
Michel Madoire \\ Bouygues Offshore
}

\section{I 口 PRÉAMBULE}

La SO.NA.RA (Société Nationale de Raffinage du Cameroun) exploite depuis une quinzaine d'années, une raffinerie située à la Pointe Limboh (nord de la côte du Cameroun).

Cette raffinerie dispose actuellement d'installations maritimes lui permettant d'effectuer le déchargement du brut à partir d'une bouée ainsi que le chargement des produits blancs à partir d'un poste situé sur une jetée constituée de caissons en béton.

La capacité d'accueil de ce poste étant limitée à des navires de 10000 tonnes, SO.NA.RA, en vue de réaliser de l'exportation, a donc décidé la construction d'un nouveau poste permettant l'accès aux navires de 30000 à 80000 tonnes. Par ailleurs, afin de supprimer les coûts de maintenance de la bouée de déchargement, SO.NA.RA a prévu d'utiliser également ce nouveau poste pour les opérations de déchargement du brut.

Suite à l'appel d'offre, organisé par TOTAL, Assistant Technique de SO.NA.RA, le 10 janvier 1994, Bouygues Offshore adjudicataire du Marché a signé le contrat relatif à l'ingénierie, fourniture, fabrication et installation de ce nouveau poste. La fabrication entièrement réalisée sur le Yard de U.I.C., filiale de Bouygues Offshore au Cameroun, a démarré en août 1994 et l'installation en janvier 1995. La livraison de l'ouvrage à SO.NA.RA sera réalisée dans le délai initial prévu au Marché, soit le 15 juillet 1995.

\section{DESCRIPTION SOMMAIRE DES OU- VRAGES}

Le poste, situé en mer ouverte à $200 \mathrm{~m}$ de la jetée existante, est constitué d'une sous-structure tubulaire en acier type « jacket » supportant un pont métallique sur lequel sont fixés tous les équipements de service et d'exploitation.

Deux ducs d'Albe d'accostage situés à l'avant de la plate-forme et 8 ducs d'Albe d'amarrage, répartis de part et d'autre de celle-ci, assurent l'ensemble des fonctions nécessaires à l'approche et à la tenue à poste des navires.

Des passerelles de lamanage permettent l'accès, à partir de la plate-forme, à chacun des ducs d'Albe.

Enfin, un viaduc de liaison constitué de cinq travées métalliques, autorise l'accès des véhicules de service à la plate-forme à partir de la jetée existante.
Les équipements principaux installés sur la plate-forme sont :

- six bras de chargement/déchargement,

- une tour avec passerelle d'accès au navire,

- une grue de service,

- un local technique ainsi que tous les équipements de contrôle et de sécurité.

Les quantités principales mises en œuvre hors équipements sont :

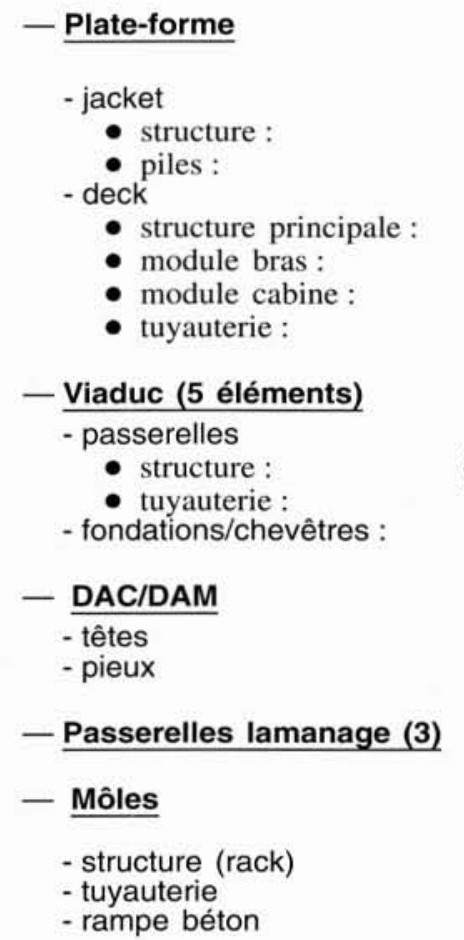

$97 \mathrm{t}$

$95 \mathrm{t}$

$97 \mathrm{t}$

$57 \mathrm{t}$

$21 \mathrm{t}$

$60 \mathrm{t}$

$192 \mathrm{t}$

$235 \mathrm{t}$

$329 \mathrm{t}$

$140 \mathrm{t}$

$262 \mathrm{t}$

$537 \mathrm{t}$

$65 \mathrm{t}$

$472 \mathrm{t}$

$68 \mathrm{t}$

$96 \mathrm{t}$

$6 \mathrm{t}$

$90 \mathrm{t}$ $102 \mathrm{~m}^{3}$

$1859 t$
TOTAL (hors béton et équipements)

\section{III — INGÉNIERIE}

L'ingénierie regroupant l'ensemble des disciplines habituelles relatives aux installations pétrolières, a été réalisée 


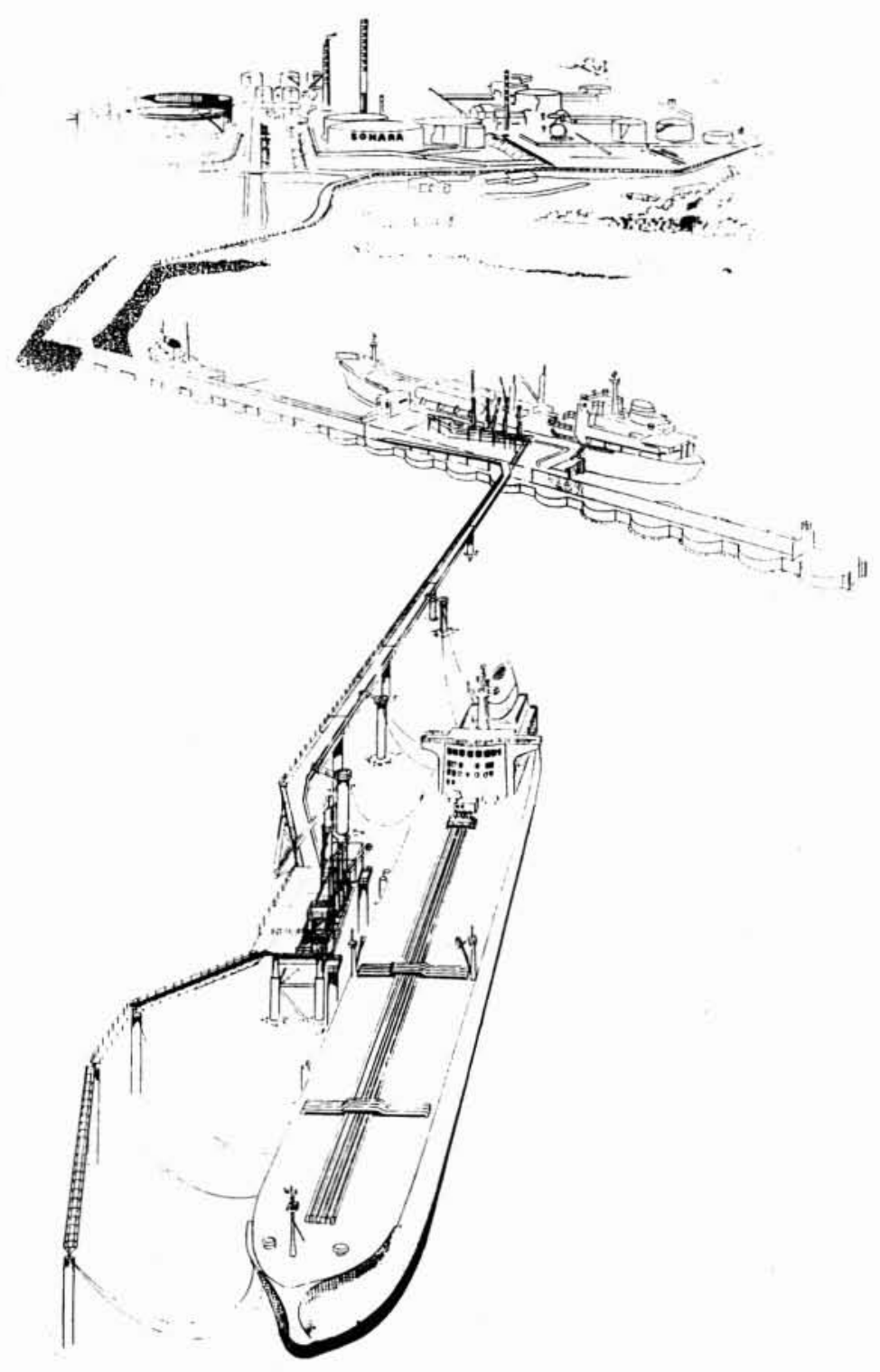

1. Vue d'artiste avec installations nouvelles en premier plan.

dans son intégralité par Bouygues Offshore, à l'exception des études d'amarrage dont une partie a été réalisée par SOGREAH.

L'intervention de SOGREAH constituait en fait un prolongement de l'étude initiale de faisabilité, réalisée pour le compte de SO.NA.RA par ce même organisme, lors de la phase d'élaboration du concept.

Cette étude, réalisée en houle régulière à l'aide du logiciel Simbad, avait pour but de définir les positions de ducs d'Albe, ainsi que les dispositions et prétensions des amarres pour toute la gamme des navires de 30000 à 80000 tonnes.

L'autre aspect de l'étude consistait à déterminer les conditions d'environnement limites à partir desquelles les tensions d'amarres et/ou les déplacements correspondants des navires devenaient supérieurs aux valeurs admissibles.

Les résultats de cette étude ont montré que, pour des houles de hauteur significative inférieure ou égale à $2,70 \mathrm{~m}$ et des périodes significatives au plus égales à $20 \mathrm{~s}$ pour le
80000 tonnes et $13 \mathrm{~s}$ pour le 30000 tonnes, les navires pouvaient opérer en toute sécurité et avec des amplitudes de mouvements très inférieures aux débattements admissibles des bras de chargement.

Une étude complémentaire en houle irrégulière réalisée par Bouygues Offshore à l'aide du logiciel Ariane dans le but de vérifier les effets éventuels basses fréquences, a confirmé les résultats de l'étude initiale.

\section{IINSTALLATION}

En raison des houles dont la période atteint fréquemment 10 à $12 \mathrm{~s}$ pour des amplitudes rarement inférieures à $1 \mathrm{~m}$, Bouygues Offshore, afin de s'affranchir de tous les problèmes liés à l'utilisation d'un support flottant, a dès le départ pris l'option de réaliser l'ensemble des opérations d'installation avec une plate-forme auto-élévatrice. 


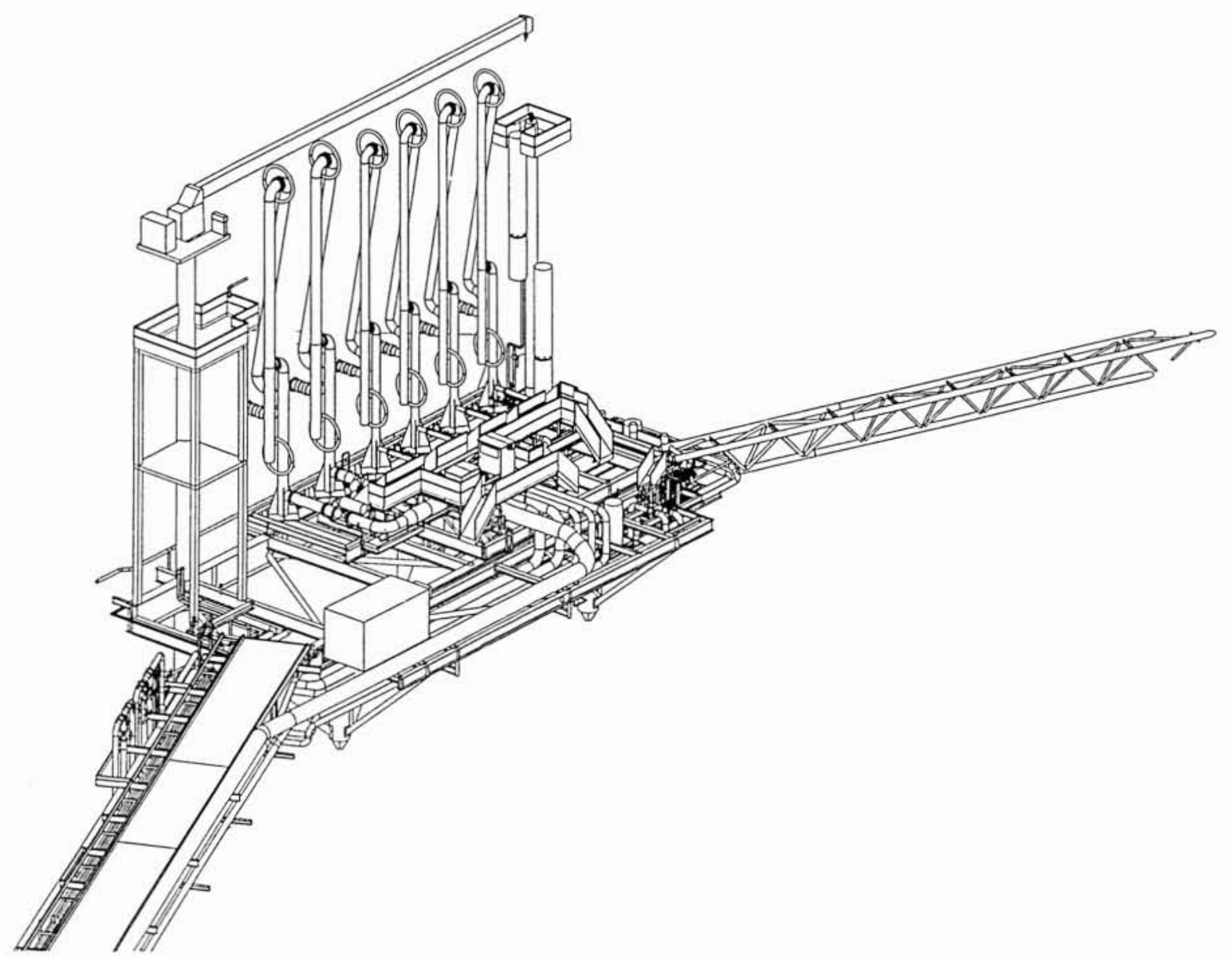

\section{Perspective de la plate-forme principale en 3D sur logiciel CAO/DAO Intergraph.}

La plate-forme utilisée, la GEM 159 de $52 \mathrm{~m} \times 24 \mathrm{~m}$, est équipée de 8 piles $1800 \emptyset$ de $68 \mathrm{~m}$ de longueur, dont le déplacement est assuré par des vérins Delong.

Cette plate-forme est équipée d'une grue MANITOWOC 4100 avec ringer, qui lui donne, pour un rayon utile de l'ordre de $23 \mathrm{~m}$, une capacité maximale de levage de $120 \mathrm{t}$

Afin de rester dans les limites de capacité utile de la grue, cette contrainte d'installation a donc été intégrée dès le début de la phase d'ingénierie de façon à obtenir un découpage de l'ouvrage en sous-ensembles de poids maximal 110 t. Par ailleurs, afin de limiter la durée et donc les coûts d'intervention sur site, il a également été pris l'option, en début de projet, de préinstaller la totalité des tuyauteries de chaque sous-ensemble de façon à ne plus avoir que les connexions à réaliser sur site.

Ce sont ces deux critères, en particulier pour le viaduc de liaison, qui ont conduit à optimiser les travées à une longueur de $40 \mathrm{~m}$.

La superstructure de la plate-forme pour répondre à ces mêmes impératifs, a dû être découpée en trois parties :

- un ensemble de 101 tonnes, constitué essentiellement de toute la structure porteuse et incluant l'unité de purge,

- un module «bras de chargement " de 87 tonnes, comportant les embases de bras et la part principale de tuyauterie,
- un module «local technique" de 31 tonnes comportant une part mineure de tuyauterie.

Toutes les autres parties de cet ouvrage étant d'un poids inférieur, voire très inférieur à la limite ci-dessus, n'ont donc pas nécessité de dispositions particulières.

Le sous-sol marin, situé entre -12 et - 17 mètres, étant constitué d'une couche sédimentaire d'épaisseur 0 à $16 \mathrm{~m}$ de caractéristiques très médiocres, il a donc été nécessaire de traverser toute cette couche, afin de réaliser un ancrage des pieux par forage et cimentation dans les sous-couches en basalte.

La foreuse utilisée, une WIRTH B6, était équipée d'un train de forage $\varnothing 1650$ pour l'ensemble des pieux, tous de diamètre 60 ", à l'exception des pieux de la plate-forme et du quadripode de blocage du viaduc en $\emptyset 40 "$.

Dans les zones avec sédiments, un tubage en 72 " était réalisé jusqu'à la couche de basalte en préalable à l'opération de forage.

Après mise en place de chaque pieu, une injection de coulis à prise rapide, était réalisée à partir de la base du pieu afin de remplir tout l'espace annulaire entre pieu / forage et ensuite pieu / tubage.

L'ancrage des pieux dans le basalte, variable en fonction des cas, était compris entre 4 et $6 \mathrm{~m}$. 